\title{
Electromagnetic Radiation in the Presence of Moving Simple Media
}

K. S. H. Lee and, and C. H. Papas

Citation: Journal of Mathematical Physics 5, 1668 (1964); doi: 10.1063/1.1704088

View online: http://dx.doi.org/10.1063/1.1704088

View Table of Contents: http://aip.scitation.org/toc/jmp/5/12

Published by the American Institute of Physics




effect has already been pointed out. ${ }^{10}$ If the point of view advanced in this note is correct, we should be investigating the relativistic generalization of the Raman spectrum of a symmetrical top. ${ }^{11}$

Rotational levels defined by equations similar to Eq. (4) have been applied to bosons of odd $S$ and to fermions of even and odd $S$, and there seems no reason why similar but more complicated equations could not be applied to atomic nuclei throughout the periodic table. This would be con-

${ }^{10}$ V. Weisskopf, Phys. Today 16, 26 (1963).

${ }^{11}$ G. Placzek and E. Teller, Z. Physik 81, 209 (1933). sistent with the philosophy of "bootstrap" calculations; vice versa, rotational levels of atomic nuclei are known to exist, and if similar techniques are applicable to states with baryon number zero and to states with baryon number of more than two hundred, it follows that rotational levels must also exist for $B=0$, and it is just a matter of computing them relativistically. This is what has been done here.

Note added in proof: This equation also leads to a fine structure at $782,789,806 \mathrm{MeV}$ and states at $1220,1653 \mathrm{MeV}$.

\title{
Electromagnetic Radiation in the Presence of Moving Simple Media
}

\author{
K. S. H. Lee and C. H. Papas \\ California Institute of Technology, Pasadena, California
}

(Received 9 June 1964)

\begin{abstract}
The radiation pattern of an arbitrary source immersed in a moving simple medium is calculated by deducing the differential equation for the potential 4 -vector in the rest frame of the source and then solving the equation in terms of a Green's function. As an illustrative example, the case where the source is an oscillating dipole is worked out in detail.
\end{abstract}

\section{INTRODUCTION}

$\mathrm{B}^{\mathrm{r}}$ $Y$ reason of its relevance to astrophysical and spatial studies, the problem of calculating the influence of a relativistic wind on the radiation properties of an arbitrary electromagnetic source is of considerable importance. In the present paper we describe a method of solving this problem for the special case where the wind is a simple medium (i.e., a homogeneous, isotropic, nondispersive dielectric) moving at uniform velocity with respect to the source.

To formulate the problem we introduce the rest frame $K$ of the source and the rest frame $K^{\prime}$ of the medium, and note that the relative motion between the source and the wind can be specified by stating that $K^{\prime}$ moves at a uniform velocity $\nabla$ with respect to $K$ or that $K$ moves at a uniform velocity $-\nabla$ with respect to $K^{\prime}$. Clearly, $K$ and $K^{\prime}$ are then inertial frames of reference and hence, in accord with Minkowski's postulate, the macroscopic Maxwell equations are covariant under the Lorentz transformation that connects the space-time coordinates of $K$ with those of $K^{\prime}$. Since the covariance of Maxwell's equations permits the combining of the vector and scalar potentials into a 4-vector, namely, the potential 4-vector, and since the field can be obtained by differentiating the potential 4-vector, knowing the potential 4-vector is tantamount to knowing the field. Thus the problem of calculating the effects of a relativistic wind on the radiation from a source amounts to one of finding the potential 4-vector in the rest frame $K$ of the source.

One may try to calculate the potential 4-vector in $K$ by first finding the potential 4 -vector in $K^{\prime}$ and then transforming the result from $K^{\prime}$ to $K$ by the proper Lorentz transformation. However, such an approach leads to difficulties, because the integral representations for the retarded potentials in $K^{\prime}$ contain the time implicitly in a complicated form. A simpler method consists of deriving the differential equation for the potential 4-vector in $K$ and then solving it by the Green's function technique. In this method the differential equation in $K$ is found by transforming the already known differential equation for the potential 4-vector in $K^{\prime}$.

Accordingly, we first find the differential equation for the potential 4-vector in the rest frame $K$ of the source, and then obtain the appropriate solution 
of the equation in terms of a Green's function. For the purpose of illustrating the method, the effect of a relativistic wind on the radiation pattern of an oscillating dipole is worked out.

\section{THE DIFFERENTIAL EQUATION FOR THE POTENTIAL 4-VECTOR}

In the rest frame $K^{\prime}$ of the wind the vector potential $\mathbf{A}^{\prime}\left(\mathbf{r}^{\prime}, t^{\prime}\right)$ and the scalar potential $\phi^{\prime}\left(\mathbf{r}^{\prime}, t^{\prime}\right)$, due to the source current density $\mathrm{J}^{\prime}\left(\mathbf{r}^{\prime}, t^{\prime}\right)$ and the source charge density $\rho^{\prime}\left(\mathbf{r}^{\prime}, t^{\prime}\right)$, obey the inhomogeneous wave equations

$$
\begin{aligned}
& \left(\nabla^{\prime 2}-\frac{n^{\prime 2}}{c^{2}} \frac{\partial^{2}}{\partial t^{\prime 2}}\right) \mathbf{A}^{\prime}=-\mu^{\prime} \mathrm{J}^{\prime}, \\
& \left(\nabla^{\prime 2}-\frac{n^{\prime 2}}{o^{2}} \frac{\partial^{2}}{\partial t^{\prime 2}}\right) \phi^{\prime}=-\frac{1}{\epsilon} \rho^{\prime},
\end{aligned}
$$

where $\mu^{\prime}$ and $\epsilon^{\prime}$ are the permeability and the dielectric constant of the simple medium and $n^{\prime}\left[=c\left(\mu^{\prime} \epsilon^{\prime}\right)^{\frac{1}{2}}\right]$ is its index of refraction. (In this section, primed quantities are referred to $K^{\prime}$ and unprimed ones to $K$.) It clearly follows from these equations that the potential 4-vector $A_{\alpha}^{\prime}=\left[\mathbf{A}^{\prime},(i / c) \phi^{\prime}\right]$ satisfies the equation

$$
\left[1+\kappa 0^{2} \delta_{4 \alpha}\right] L^{\prime} A_{\alpha}^{\prime}=-\mu^{\prime} J_{\alpha}^{\prime} \quad(\alpha=1,2,3,4),
$$

where $\delta_{4 \alpha}$ is the Kronecker delta, $\kappa$ is a shorthand for $\left(n^{\prime 2}-1\right) / c^{2}$, and $L^{\prime}$ is the operator $\nabla^{\prime 2}-$ $\left(n^{\prime 2} / c^{2}\right) \partial^{2} / \partial t^{\prime 2}$. The source term on the right side is the current density 4-vector $J_{\alpha}^{\prime}=\left(\mathrm{J}^{\prime}, i c \rho^{\prime}\right)$.

To transform Eq. (2.2) to the reference frame $K$ we note that since $A_{\alpha}^{\prime}$ and $J_{\alpha}^{\prime}$ are 4-vectors they are related to their counterparts $A_{\beta}, J_{\beta}$ in $K$ by

$$
A_{\alpha}^{\prime}=a_{\alpha \beta} A_{\beta}, \quad J_{\alpha}^{\prime}=a_{\alpha \beta} J_{\beta},
$$

where $a_{\alpha \beta}$ are the coefficients of the proper Lorentz transformation that carries $K$ into $K^{\prime}$. Furthermore, by writing $L^{\prime}$ as

$$
L^{\prime}=\nabla^{\prime 2}-\frac{1}{a^{2}} \frac{\partial^{2}}{\partial t^{\prime 2}}-\kappa \frac{\partial^{2}}{\partial t^{\prime 2}},
$$

and then noting that the first two terms constitute an invariant and that the third term transforms according to the rule

$$
\partial / \partial t^{\prime}=\gamma(\partial / \partial t)+\gamma \mathbf{v} \cdot \nabla,
$$

where $\gamma=\left(1-v^{2} / c^{2}\right)^{-\frac{1}{2}}$, we see that $L^{\prime}$ transforms into

$$
L=\nabla^{2}-\frac{1}{a^{2}} \frac{\partial^{2}}{\partial t^{2}}-\kappa \gamma^{2}\left(v \cdot \nabla+\frac{\partial}{\partial t}\right)^{2} .
$$

In view of Eqs. (2.3) and (2.6) it is clear that
Eq. (2.2) becomes

$$
\left[1+\kappa c^{2} \delta_{4 \alpha}\right] a_{\alpha \beta} L A_{\beta}=-\mu^{\prime} a_{\alpha \beta} J_{\beta} .
$$

Applying the orthogonality relation $a_{\alpha{ }} a_{\alpha \beta}=\delta_{\nu \beta}$ to this equation, we then obtain

$$
L A_{\nu}=-\mu^{\prime} J_{\nu}+\left(\kappa c^{2} / n^{\prime 2}\right) \mu^{\prime} a_{4,} a_{4 \beta} J_{\beta},
$$

which in terms of the velocity 4-vector $U_{\nu}=(\gamma \nabla, i \gamma c)$ yields

$$
L A_{\nu}=-\mu^{\prime} J_{\nu}-\frac{\kappa}{n^{\prime 2}} \mu^{\prime} J_{\alpha} U_{\alpha} U_{\nu} .
$$

In three dimensions Eq. (2.9) takes the form

$$
\begin{aligned}
& {\left[\nabla^{2}-\frac{1}{o^{2}} \frac{\partial^{2}}{\partial t^{2}}-\kappa \gamma^{2}\left(\frac{\partial}{\partial t}+\nabla \cdot \nabla\right)^{2}\right] \mathrm{A}} \\
& =-\mu^{\prime} \mathrm{J}-\frac{\mu^{\prime} \kappa}{n^{\prime 2}} \gamma^{2} \nabla \nabla \cdot \mathrm{J}+\frac{\mu^{\prime} \kappa 0^{2}}{n^{\prime 2}} \gamma^{2} \rho \nabla \\
& {\left[\nabla^{2}-\frac{1}{o^{2}} \frac{\partial^{2}}{\partial t^{2}}-\kappa \gamma^{2}\left(\frac{\partial}{\partial t}+\nabla \cdot \nabla\right)^{2}\right]_{\phi}} \\
& =-o^{2} \mu^{\prime}\left(1-\frac{\kappa c^{2}}{n^{\prime 2}} \gamma^{2}\right) \rho-\frac{\mu^{\prime} \kappa c^{2}}{n^{\prime 2}} \gamma^{2} \nabla \cdot \mathrm{J}
\end{aligned}
$$

Thus we see that the inhomogeneous wave equations (2.1) for $\mathbf{A}^{\prime}, \phi^{\prime}$ in $K^{\prime}$ transform into Eqs. (2.10) for A, $\phi$ in $K .^{I}$

\section{GREEN'S FUNCTION REPRESENTATION OF RADIATION FIELD}

To solve Eqs. (2.10) we represent A, $\phi, \rho$, and J by the Fourier integrals

$$
\begin{aligned}
& \mathbf{A}(\mathbf{r}, t)=\int_{-\infty}^{\infty} \mathbf{A}_{\omega}(\mathbf{r}) e^{-i \omega t} d \omega, \\
& \phi(\mathbf{r}, t)=\int_{-\infty}^{\infty} \phi_{\omega}(\mathbf{r}) e^{-i \omega t} d \omega, \\
& \rho(\mathbf{r}, t)=\int_{-\infty}^{\infty} \rho_{\omega}(\mathbf{r}) e^{-i \omega t} d \omega, \\
& \mathbf{J}(\mathbf{r}, t)=\int_{-\infty}^{\infty} \mathbf{J}_{\omega}(\mathbf{r}) e^{-i \omega t} d \omega,
\end{aligned}
$$

and note that the continuity equation $\nabla \cdot \mathrm{J}(\mathbf{r}, t)=$ $-(\partial / \partial t) \rho(\mathbf{r}, t)$ yields

$$
\nabla \cdot \mathrm{J}_{\omega}(\mathbf{r})=i_{\omega \rho_{\omega}}(\mathbf{r})
$$

Substituting representations (2.11) into Eqs. (2.10) and using Eq. (2.12) we see that $\mathbf{A}_{\omega}(\mathbf{r})$ and $\phi_{\omega}(\mathbf{r})$

1 Actually, the same result can be achieved by using the tensor form of Maxwell's equations as the point of departure. See J. M. Jauch and K. M. Watson, Phys. Rev. 74, 950, 1485 
satisfy

$$
\begin{array}{r}
{\left[\nabla^{2}+\omega^{2} / 0^{2}-\kappa \gamma^{2}(\mathbf{v} \cdot \nabla-i \omega)^{2}\right] \phi_{\omega}(\mathbf{r})} \\
=\mathbf{M} \cdot \mathrm{J}_{\omega}(\mathbf{r}), \\
{\left[\nabla^{2}+\omega^{2} / c^{2}-\kappa \gamma^{2}(\mathbf{v} \cdot \nabla-i \omega)^{2}\right] \mathbf{A}_{\omega}(\mathbf{r})} \\
=\mathbf{N} \cdot \mathrm{J}_{\omega}(\mathbf{r}),
\end{array}
$$

where the vector $\mathbf{M}$ and the dyadic $\mathbf{N}$ are given by

$$
\begin{gathered}
\mathbf{M}=-\frac{\sigma^{2} \mu^{\prime}}{i \omega}\left(1-\frac{\kappa c^{2}}{n^{\prime 2}} \gamma^{2}\right) \nabla-\frac{\mu^{\prime} \kappa c^{2}}{n^{\prime 2}} \gamma^{2} \nabla, \\
\mathbf{N}=-\mu^{\prime} \mathrm{U}-\frac{\mu^{\prime} \kappa}{n^{\prime 2}} \gamma^{2} \nabla \nabla+\frac{\mu^{\prime} \kappa c^{2}}{i \omega n^{\prime 2}} \gamma^{2} \nabla \nabla,
\end{gathered}
$$

and $U$ is the unit dyadic.

From the linearity of these equations it is clear that their solutions are

$$
\begin{aligned}
& \boldsymbol{\phi}_{\omega}(\mathbf{r})=-\mathbf{M} \cdot \int \mathrm{J}_{\omega}\left(\mathbf{r}^{\prime}\right) G_{\omega}\left(\mathbf{r}, \mathbf{r}^{\prime}\right) d V^{\prime}, \\
& \mathbf{A}_{\omega}(\mathbf{r})=-\mathbf{N} \cdot \int \mathbf{J}_{\omega}\left(\mathbf{r}^{\prime}\right) G_{\omega}\left(\mathbf{r}, \mathbf{r}^{\prime}\right) d V^{\prime},
\end{aligned}
$$

provided the function $G_{\omega}\left(r, r^{\prime}\right)$ is a solution of

$$
\begin{array}{r}
{\left[\nabla^{2}+\omega^{2} / o^{2}-\kappa \gamma^{2}(\mathbf{v} \cdot \nabla-i \omega)^{2}\right] G_{\omega}\left(\mathbf{r}, \mathbf{r}^{\prime}\right)} \\
=-\delta\left(\mathbf{r}-\mathbf{r}^{\prime}\right) .
\end{array}
$$

The solution of (2.17) is the Green's function

$$
\begin{aligned}
& G_{\omega}\left(\mathbf{r}, \mathbf{r}^{\prime}\right) \\
& =\frac{1}{8 \pi^{3}} \iiint_{-\infty}^{\infty} \frac{e^{i \mathbf{k} \cdot\left(\mathbf{r}-\mathbf{r}^{\prime}\right)}}{k^{2}-\omega^{2} / c^{2}-\kappa \gamma^{2}(\mathbf{v} \cdot \mathbf{k}-\omega)^{2}} d^{3} k .
\end{aligned}
$$

From a knowledge of the Green's function $G_{\omega}\left(\mathbf{r}, \mathbf{r}^{\prime}\right)$, the potentials $\phi_{\omega}(\mathbf{r})$ and $A_{\omega}(\mathrm{r})$, due to an arbitrary current density $J_{\omega}(r)$, can be found from relations (2.16). In turn, the electric vector $\mathbf{E}_{\omega}(\mathbf{r})$ and the magnetic vector $B_{\omega}(r)$ can be found from a knowledge of $\phi_{\omega}(\mathbf{r})$ and $\mathbf{A}_{\omega}(\mathbf{r})$ by using the relations

$$
\mathbf{E}_{\omega}=-\nabla \phi_{\omega}+i \omega \mathbf{A}_{\omega}, \quad \mathbf{B}_{\omega}=\nabla \times \mathbf{A}_{\omega} .
$$

Thus the calculation of the electromagnetic field $\mathbf{E}_{\omega}, \mathbf{B}_{\omega}$ radiated by the current $J_{\omega}(r)$ hinges on the evaluation of the Green's function (2.18).

\section{EVALUATION OF THE GREEN'S FUNCTION}

Without loss of generality we choose the $z$ axis of the reference frame $K$ to be parallel to the velocity $\mathbf{v}$ of the moving medium. With this choice the integral representation for the Green's function becomes

$$
\begin{aligned}
& G_{\omega}\left(\mathbf{r}, \mathbf{r}^{\prime}\right) \\
& \quad=\frac{1}{8 \pi^{3}} \iiint_{-\infty}^{\infty} \frac{e^{i k_{x}\left(x-x^{\prime}\right)} e^{i k_{y}\left(y-y^{\prime}\right)} e^{i k_{z}\left(z-z^{\prime}\right)}}{k^{2}-k_{0}^{2}-\kappa \gamma^{2}\left(v k_{z}-\omega\right)^{2}} d k_{x} d k_{y} d k_{z},
\end{aligned}
$$

where $k_{0}^{2}=\omega^{2} / c^{2}$. In preparation for the $k_{z}$ integration we write the denominator of the integrand in the factored form

$$
\begin{aligned}
& k^{2}-k_{0}^{2}-\kappa \gamma^{2}\left(v k_{z}-\omega\right)^{2} \\
& \quad=\gamma^{2}\left(1-{n^{\prime 2}}^{2} \beta^{2}\right)\left(k_{z}-\tau_{+}\right)\left(k_{z}-\tau_{-}\right),
\end{aligned}
$$

where

$$
\tau_{ \pm}=\frac{-k_{0} \beta \gamma^{2}\left(n^{\prime 2}-1\right) \pm\left[n^{\prime 2} k_{n}^{2}-\gamma^{2}\left(1-n^{\prime 2} \beta^{2}\right)\left(k_{x}^{2}+k_{y}^{2}\right)\right]^{\frac{1}{2}}}{\gamma^{2}\left(1-n^{\prime 2} \beta^{2}\right)}
$$

always lie below both $\tau_{+}$and $\tau_{-}$. In any case the contour is closed in the upper half-plane for $z-z^{\prime}>0$ and in the lower half-plane for $z-z^{\prime}<0$.

Accordingly, when $n^{\prime} \beta<1$ and $z-z^{\prime}>0$, expression (2.20) becomes

$$
\begin{aligned}
& G_{\omega}\left(\mathbf{r}, \mathbf{r}^{\prime}\right)=\frac{1}{8 \pi^{3}} \frac{2 \pi i}{\gamma^{2}\left(1-n^{\prime 2} \beta^{2}\right)} \\
& \quad \times \iint_{-\infty}^{\infty} \frac{e^{i \tau+\left(z-z^{\prime}\right)} e^{i k_{s}\left(x-x^{\prime}\right)} e^{i k_{y}\left(y-y^{\prime}\right)}}{\tau_{+}-\tau_{-}} d k_{x} d k_{y} .
\end{aligned}
$$

Introducing the polar coordinates $(s, \chi)$ by the relations $k_{x}=s \cos \chi, k_{y}=s \sin \chi$ and integrating with respect to $\chi$ from $\chi=0$ to $\chi=2 \pi$, we get

$$
G_{\omega}\left(\mathbf{r}, \mathbf{r}^{\prime}\right)=\frac{a}{4 \pi} e^{-i b k\left(z-z^{\prime}\right)} \int_{0}^{\infty} \frac{J_{0}\left\{s\left[\left(x-x^{\prime}\right)^{2}+\left(y-y^{\prime}\right)^{2}\right]^{\frac{1}{2}}\right\} \exp \left[i a\left(k^{2}-s^{2}\right)^{\frac{3}{2}}\left(z-z^{\prime}\right)\right]}{\left(s^{2}-k^{2}\right)^{\frac{1}{2}}} s d s,
$$


where

$$
\begin{gathered}
a=\left[\gamma\left(1-{n^{\prime}}^{2} \beta^{2}\right)^{\frac{1}{2}}\right]^{-1}, \\
b=a \beta \gamma^{2}\left[\left({n^{\prime}}^{2}-1\right) / n^{\prime}\right], \quad k=a n^{\prime} k_{0} .
\end{gathered}
$$

Then by evaluating the integral we have

$$
\begin{aligned}
& G_{\omega}\left(\mathbf{r}, \mathbf{r}^{\prime}\right)=\frac{a}{4 \pi} e^{-i b k\left(z-z^{\prime}\right)} \\
& \quad \times \frac{\exp \left\{i k\left[\left(x-x^{\prime}\right)^{2}+\left(y-y^{\prime}\right)^{2}+a^{2}\left(z-z^{\prime}\right)^{2}\right]^{\frac{1}{b}}\right\}}{\left[\left(x-x^{\prime}\right)^{2}+\left(y-y^{\prime}\right)^{2}+a^{2}\left(z-z^{\prime}\right)^{2}\right]^{\frac{1}{3}}} .
\end{aligned}
$$

It turns out that this result is valid for $z-z^{\prime}<0$ also. Hence, when $n^{\prime} \beta<1$ we see that in general

$G_{\omega}\left(\mathbf{r}, \mathbf{r}^{\prime}\right)=\frac{a}{4 \pi} \exp \left[-i\left(\frac{b k}{v}\right) \mathbf{v} \cdot\left(\mathbf{r}-\mathbf{r}^{\prime}\right)\right]$

$$
\times \frac{\exp \left\{i k\left[\left(\mathbf{r}-\mathbf{r}^{\prime}\right)^{2}+\frac{a^{2}-1}{v^{2}}\left[\mathbf{v} \cdot\left(\mathbf{r}-\mathbf{r}^{\prime}\right)\right]^{2}\right]^{\frac{1}{2}}\right\}}{\left[\left(\mathbf{r}-\mathbf{r}^{\prime}\right)^{2}+\frac{a^{2}-1}{v^{2}}\left[\mathbf{v} \cdot\left(\mathbf{r}-\mathbf{r}^{\prime}\right)\right]^{2}\right]^{\frac{1}{2}}} .
$$

When $n^{\prime} \beta>1$, expression (2.20) yields

$$
\begin{aligned}
& G_{\omega}\left(\mathbf{r}, \mathbf{r}^{\prime}\right)=\frac{a}{4 \pi^{2}} e^{i b k\left(z-z^{\prime}\right)} \\
& \quad \times \int_{0}^{\infty} \int_{0}^{2 \pi} \frac{\sin \left[a\left(z-z^{\prime}\right)\left(s^{2}+k^{2}\right)^{\frac{3}{2}}\right]}{\left(s^{2}+k^{2}\right)^{\frac{1}{2}}} \\
& \quad \times \exp \left[i s\left(x-x^{\prime}\right) \cos \chi+i s\left(y-y^{\prime}\right) \sin \chi\right] s d \chi d s
\end{aligned}
$$

for $z-z^{\prime}>0$, and

$$
G_{\omega}\left(\mathbf{r}, \mathbf{r}^{\prime}\right)=0
$$

for $z-z^{\prime}<0$. Here $a=\left[\gamma\left(n^{\prime 2} \beta^{2}-1\right)^{\frac{1}{3}}\right]$. Carrying out the $\chi$ integration we get

$$
G_{\omega}\left(\mathbf{r}, \mathbf{r}^{\prime}\right)=\frac{a}{2 \pi} e^{i b k\left(z-z^{\prime}\right)} \int_{0}^{\infty} \frac{J_{0}\left\{s\left[\left(x-x^{\prime}\right)^{2}+\left(y-y^{\prime}\right)^{2}\right]^{\frac{3}{3}}\right\} \sin \left[a\left(z-z^{\prime}\right)\left(s^{2}+k^{2}\right)^{\frac{1}{3}}\right]}{\left(s^{2}+k^{2}\right)^{\frac{1}{2}}} s d s
$$

which in turn yields

$$
\begin{aligned}
& G_{\omega}\left(\mathbf{r}, \mathbf{r}^{\prime}\right)=\frac{a}{2 \pi} e^{i b k\left(z-z^{\prime}\right)} \\
& \quad \times \frac{\cos \left\{k\left[-\left(x-x^{\prime}\right)^{2}-\left(y-y^{\prime}\right)^{2}+a^{2}\left(z-z^{\prime}\right)^{2}\right]^{\frac{1}{3}}\right\}}{\left[-\left(x-x^{\prime}\right)^{2}-\left(y-y^{\prime}\right)^{2}+a^{2}\left(z-z^{\prime}\right)^{2}\right]^{\frac{1}{2}}}
\end{aligned}
$$

for

and

$$
z-z^{\prime}>\left[\left(x-x^{\prime}\right)^{2}+\left(y-y^{\prime}\right)^{2}\right]^{\frac{1}{2}} a^{-1},
$$

$$
G_{\omega}\left(\mathbf{r}, \mathbf{r}^{\prime}\right)=0
$$

for $z-z^{\prime}<\left[\left(x-x^{\prime}\right)^{2}+\left(y-y^{\prime}\right)^{2}\right]^{\frac{1}{2}} a^{-1}$. This shows that when $n^{\prime} \beta>1$ the Green's function is different from zero only within a conical region in the direction of the wind (Fig. 1). The half-angle $\theta_{0}$ of the cone is given by

$$
\tan \theta_{0}=a=\left[\gamma\left(n^{\prime 2} \beta^{2}-1\right)^{\frac{1}{1}}\right]^{-1}
$$

\section{RADIATION FIELD OF AN OSCILLATING DIPOLE}

Let us now consider the far-zone field of an oscillating dipole of frequency $\omega$ for the case where $n^{\prime} \beta<1$. Let the dipole be located at the origin of a spherical coordinate system $(r, \theta, \phi)$. If $\mathrm{p}$ is its dipole moment, then $\mathbf{J}_{\omega}\left(\mathbf{r}^{\prime}\right)=-i \omega \mathbf{p} \delta\left(\mathbf{r}^{\prime}\right)$. Accordingly, with the aid of expression (2.27) we see that

$$
\begin{array}{r}
\int \mathrm{J}_{\omega}\left(\mathbf{r}^{\prime}\right) G_{\omega}\left(\mathbf{r}, \mathbf{r}^{\prime}\right) d V^{\prime}=-i \omega \mathrm{p} a e^{-i b k r \cos \theta} \\
\times \frac{\exp \left[i k r \left(\sin ^{2}\right.\right.}{4 \pi r\left(\sin ^{2} \theta\right.} \frac{\left.\left.\theta+a^{2} \cos ^{2} \theta\right)^{\frac{k}{2}}\right]}{\left.a^{2} \cos ^{2} \theta\right)^{\frac{1}{2}}}
\end{array}
$$

where $\theta$ is the angle between $\nabla$ and $\mathbf{r}$. Substituting expression (2.34) into Eqs. (2.16) one can obtain the scalar and vector potentials of the dipole, and then from these potentials can deduce by use of the first of Eqs. (2.19) the electric field of the dipole.

Assuming that $\mathbf{p}$ is perpendicular to $\mathbf{v}$, i.e., $\mathbf{p}=\mathbf{e}_{x} p$ and $\mathbf{v}=\mathbf{e}_{z} v$, we find that the far-zone electric field is given by

$$
\begin{gathered}
E_{\theta}=\omega^{2} \mu^{\prime} p \frac{a^{3}}{\sin ^{2} \theta+a^{2} \cos ^{2} \theta} \cos \theta \cos \phi e^{-i b k r \cos \theta} \\
\times \frac{\exp \left[i k r\left(\sin ^{2} \theta+a^{2} \cos ^{2} \theta\right)^{\frac{1}{2}}\right]}{4 \pi r\left(\sin ^{2} \theta+a^{2} \cos ^{2} \theta\right)^{\frac{1}{2}}},
\end{gathered}
$$$$
E_{\phi}=-\omega^{2} \mu^{\prime} p a \sin \phi e^{-i b k r \cos \theta}
$$

$$
\begin{gathered}
\times \frac{\exp \left[i k r\left(\sin ^{2} \theta+a^{2} \cos ^{2} \theta\right)^{\frac{1}{2}}\right]}{4 \pi r\left(\sin ^{2} \theta+a^{2} \cos ^{2} \theta\right)^{\frac{1}{2}}}, \\
E_{r}=0
\end{gathered}
$$

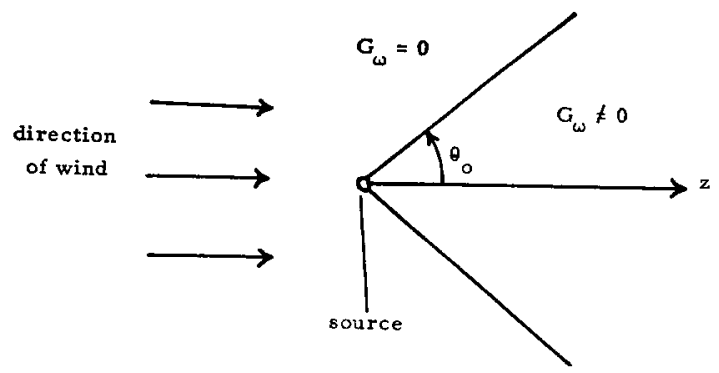

FIG. 1. Conical region where Green's function is not identically zero. 


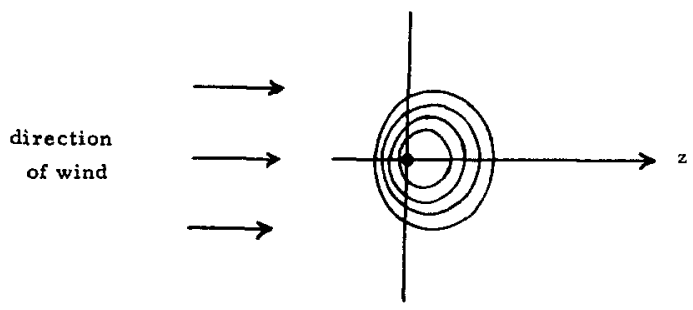

Fig. 2. Surfaces of constant phase dragged by wind.

where, as before

$a=\frac{1}{\gamma\left(1-n^{\prime 2} \beta^{2}\right)^{\frac{1}{2}}}, \quad b=a \beta \gamma^{2} \frac{n^{\prime 2}-1}{n^{\prime}}, \quad k=a n^{\prime} \frac{\omega}{c}$.

In the case where $\mathbf{p}$ is parallel to $\nabla$, i.e., $\mathbf{p}=\mathbf{e}_{x} p$ and $\mathrm{v}=\mathbf{e}_{z} v$, we find that

$$
\begin{gathered}
E_{\theta}=-\omega^{2} \mu^{\prime} p \frac{a^{3}}{\left(\sin ^{2} \theta+a^{2} \cos ^{2} \theta\right)^{\frac{1}{2}} \sin \theta e^{-i b k r \cos \theta}} \\
\times \frac{\exp \left[i k r\left(\sin ^{2} \theta+a^{2} \cos ^{2} \theta\right)^{\frac{3}{3}}\right]}{4 \pi r\left(\sin ^{2} \theta+a^{2} \cos ^{2} \theta\right)^{\frac{1}{2}}}, \\
E_{\phi}=E_{r}=0 .
\end{gathered}
$$

In examining the above expressions for the far- zone field components we see that the phase of each is given by

$$
\psi=-b k z+k\left(x^{2}+y^{2}+a^{2} z^{2}\right)^{\frac{3}{2}}-\omega t .
$$

The surfaces of constant phase, i.e., surfaces defined by $\psi=$ const, are oblate spheroids. If the medium were not moving, the surfaces would be spheres. That is, the motion of the medium contracts these spheres in the direction of motion and thus deforms them into oblate spheroids. The axis of rotational symmetry of each spheroid passes through the source and is parallel to the direction of the wind. As time increases, a spheroidal surface of constant phase expands and is dragged by the wind (Fig. 2).

For the complementary case where $n^{\prime} \beta>1$ the Green's function that enters the calculation is given by Eq. (2.31), and the far-zone radiation field can be found in much the same way as in the case where $n^{\prime} \beta<1$.

\section{ACKNOWLEDGMENT}

The authors are grateful to the U. S. Air Force Office of Scientific Research for supporting this research.

\title{
High-Field Magnetoresistance of Inhomogeneous Semiconductors and Plasmas. II. Two-Dimensional Inhomogeneity Distributions
}

\author{
J. A. Morrison And H. L. Frisch \\ Bell Telephone Laboratories, Incorporated, Murray Hill, New Jersey \\ (Received 11 February 1964; final manuscript received 24 July 1964)
}

\begin{abstract}
We extend in this paper the treatment of the high field magnetoresistance of a previously described classical model of a semiconductor (plasma) containing a two-dimensional distribution of inhomogeneities. The basic assumptions on the classical model are that the scale of the inhomogeneities is large compared to the mean thermal wavelength of an electron and the Landau level spacing is large compared to $k T$. The magnetic field $\mathfrak{H}$ is taken parallel to the $z$ coordinate and the inhomogeneity distribution is characterized by a sufficiently smooth potential $\varphi(x, z)$. The 4-moment equations are solved asymptotically for large $\mathfrak{H}$, and an equivalent asymptotic solution is obtained, subject to certain mathematical assumptions, for the transport equation. The magnetoresistance is shown, in general, not to saturate, but to increase, as $\mathcal{H}^{2}$, with increasing $\mathcal{H}$.
\end{abstract}

\section{INTRODUCTION}

$\mathrm{T}$ HIS is the second in a series of papers in which we examine the possibility that the high-field nonsaturation of the magnetoresistance of a semiconductor (or a model plasma) has a classical origin in the presence in the sample of a more or less random distribution of spatial inhomogeneities. The first paper, ${ }^{1}$ hereafter referred to as I, dealt only with the special case of a stratified medium. Here we extend the theory developed in I to two-dimensional inhomogeneity distributions which vary both along the $x$ and $z$ coordinates, the latter being

1 H. L. Frisch and J. A. Morrison, Ann. Phys. 26, 181 (1964). Equations referring to this paper are prefixed with a $I$. 